\title{
Philosophiques
}

\section{Prendre les autres au sérieux : sur le débat cosmopolitisme-particularisme}

\section{María Herrera Lima}

Volume 34, numéro 1, printemps 2007

Cosmopolitisme et particularisme

URI : https://id.erudit.org/iderudit/015861ar

DOI : https://doi.org/10.7202/015861ar

Aller au sommaire du numéro

\section{Éditeur(s)}

Société de philosophie du Québec

ISSN

0316-2923 (imprimé)

1492-1391 (numérique)

Découvrir la revue

Citer cet article

Herrera Lima, M. (2007). Prendre les autres au sérieux : sur le débat cosmopolitisme-particularisme. Philosophiques, 34(1), 17-40.

https://doi.org/10.7202/015861ar
Résumé de l'article

Dans cet essai, je tente de répondre à quelques-unes des objections habituellement adressées au cosmopolitisme, en prenant en considération la perspective de ceux que cette doctrine risque d'affecter, à savoir, les immigrants. J'argue que certaines de ces objections se fondent sur des conceptions simplifiées à l'excès et empiriquement inexactes de ce que sont la communauté et l'identité culturelle. J'examine aussi en détail la controverse entourant les possibles implications autoritaristes - ou impérialistes - des propositions visant à faire d'un système moral-légal un modèle pour le monde. L'objectif général de cet article est d'explorer les conditions permettant l'établissement d'une règle de justice cosmopolitique, dans un contexte mondial marqué par des différences culturelles et une extrême inégalité dans l'accès aux ressources et au pouvoir décisionnel. Certaines questions relatives à la motivation morale sont aussi abordées. 


\title{
Prendre les autres au sérieux: sur le débat cosmopolitisme-particularisme
}

\author{
MARÍA HERRERA LIMA \\ Instituto de Investigaciones Filosóficas \\ UNAM, México
}

\begin{abstract}
RÉSUMÉ. - Dans cet essai, je tente de répondre à quelques-unes des objections habituellement adressées au cosmopolitisme, en prenant en considération la perspective de ceux que cette doctrine risque d'affecter, à savoir, les immigrants. J'argue que certaines de ces objections se fondent sur des conceptions simplifiées à l'excès et empiriquement inexactes de ce que sont la communauté et l'identité culturelle. J'examine aussi en détail la controverse entourant les possibles implications autoritaristes - ou impérialistes - des propositions visant à faire d'un système moral-légal un modèle pour le monde. L'objectif général de cet article est d'explorer les conditions permettant l'établissement d'une règle de justice cosmopolitique, dans un contexte mondial marqué par des différences culturelles et une extrême inégalité dans l'accès aux ressources et au pouvoir décisionnel. Certaines questions relatives à la motivation morale sont aussi abordées.
\end{abstract}

\section{Introduction}

L'apparition de nouvelles formes d'interdépendance, ainsi que l'accroissement des interactions sociales et économiques à l'échelle mondiale, ont élargi la portée de nos responsabilités morales. Ainsi apparaissent de nouvelles questions pour la théorie morale ${ }^{1}$ de même que de nouvelles façons d'aborder de vieux problèmes. C'est ainsi que le problème usuel de définir un "test de légitimité » pour toute conception de la justice qui aspire à une validité universelle requiert, aujourd'hui plus que jamais, de comprendre comment il serait possible d'obtenir le consentement actuel — et non seulement hypothétique de toutes les personnes concernées. Si on en appelle maintenant à une justification morale et politique - plutôt qu'exclusivement légale — de la justice, c'est parce que les règles communes de justice, si elles étaient vues comme simplement imposées, n'auraient pas la force persuasive que présuppose leur application. Il se pourrait fort bien qu'on doive renouveler notre compréhension des raisons qui motivent la moralité. Pour y arriver dans un contexte multiculturel, on doit, d'une part, examiner les désaccords normatifs qui sont reliés à des différences culturelles et, d'autre part, tenir compte des utilisations idéologiques de ces normes en tant qu'exigences posées à autrui dans des conditions d'inégalité touchant aussi bien les ressources matérielles que l'accès à l'usage de la force militaire. Dans de telles conditions, on pourrait ainsi se demander: quelles sortes de raisons faut-il pour convaincre ou persuader les

1. Comme dans le cas de la pauvreté et de l'inégalité dans le monde. Voir, par exemple, Pogge, 2001, pp. 6-24.

PHILOSOPHIQUES 34/1 — Printemps 2007, p. 17-40 
autres d'adopter des normes et des principes - tels que le respect des droits de la personne et des libertés civiles - qui sont étrangers à leurs traditions et qui, de plus, pourraient faire peser sur eux la menace de sanctions ou d'actions punitives en cas de violation de ces normes ou principes? On pourrait voir cette question comme une variation de ce que Christine Korsgaard considère comme la question normative par excellence: pourquoi devrais-je faire cela ${ }^{2}$ ?

Ce sont là des questions philosophiques, mais elles appellent une réponse relativement modeste, à savoir, une définition des conditions préalables à l'établissement de directives morales-légales. Ces conditions de légitimité pourraient correspondre aux trois critères posés par Korsgaard pour les théories normatives: a) du point de vue de l'agent, une théorie normative doit fournir « une justification des demandes que lui impose la moralité »; b) une condition de «transparence» qu'elle formule comme suit: «Une théorie morale normative doit nous permettre d'agir à la pleine lumière, ou en toute connaissance, de ce qu'est la moralité et des raisons pour lesquelles nous sommes soumis à son influence et, en même temps, de croire que nos actions sont justifiées et pourvues de sens "; et c) les réponses normatives "doivent faire appel à notre sens profond de qui nous sommes, à notre sens de l'identités ». La deuxième condition est peut-être trop contraignante pour pouvoir s'appliquer dans un contexte multiculturel et pourrait sans doute être reformulée en termes plus faibles. Mais nous devrions à tout le moins essayer de satisfaire aux conditions $a$ et $c$.

La question de la justice mondiale est aussi, évidemment, lourde d'implications politiques. Pourquoi aurait-on besoin d'un tel ordre mondial ? Comment pourrait-il être instauré ? Est-il possible de mettre en place un système de droit international et des institutions supranationales sans donner des privilèges injustifiés à certains pays et à leurs besoins particuliers ? Est-il possible de défendre une certaine idée de la démocratie et du respect des droits de la personne sans encourir le soupçon d'être les alliés de ceux qui, en raison de leurs intérêts particuliers, prétendent la défendre - particulièrement en usant de la force militaire? Avec ces questions présentes à l'esprit, on peut encore se demander si, et comment, malgré tout, une version cosmopolitique de l'universalisme moral-légal peut constituer une théorie viable de la justice mondiale.

On doit d'abord remarquer que le cosmopolitisme désigne un large éventail de positions légales, politiques et philosophiques ${ }^{4}$. Historiquement dérivé de la reconnaissance de la diversité culturelle chez les anciens, il constitue le premier énoncé explicite de l'idée voulant qu'une humanité commune, sousjacente à cette diversité, soit la source d'obligations morales — en particulier,

2. Korsgaard, 1996, p. 16.

3. Ibid, pp. 16-17.

4. Il pourrait être défini, par exemple, en termes de communauté morale universelle, de loi internationale ou de marché mondial. Voir Kleingeld, 1999. 
le devoir d'hospitalité envers les étrangers. Il est ensuite associé à la curiosité et à l'intérêt, suscités par les voyages, à l'endroit de la nouveauté et de la différence, et, dans la modernité précoce, à certaines conceptions de la tolérance politique. Mais, sans aucun doute, ce fut le fameux essai de Kant, Vers la paix perpétuelle ${ }^{5}$, qui a établi en son temps la compréhension du cosmopolitisme en tant qu'ordre politique juste allant bien au delà des traités de non-agression entre États, et qui a fait du cosmopolitisme une loi universelle pour l'humanité ${ }^{6}$. Je n'aborderai pas directement le projet kantien dans cet article; je me concentrerai plutôt sur les conditions qui président à l'acceptation généralisée d'une justice minimale à l'échelle mondiale.

Dans les débats récents, la plupart des idées du cosmopolitisme ancien sont reprises dans la perspective des problèmes qui résultent de la nouvelle situation mondiale. Il n'est guère surprenant que certains des thèmes anciens réapparaissent en ces temps de réajustement des zones d'influences politiques et militaires des grandes puissances mondiales, de même qu'avec l'apparition de nouvelles formes d'organisation économique et le développement des communications de masse à l'échelle mondiale. D'une part, la nouvelle situation nous force à définir de nouveaux mécanismes pour la coordination sociale des intérêts collectifs aux niveaux nationaux et internationaux. Cette tâche suppose la recherche de solutions viables à de nouvelles formes de conflits qui opposent les coutumes, les valeurs et les intérêts qui ont cours à l'échelon local à ceux des puissances mondiales, des corporations transnationales et des institutions supranationales. D'autre part, on observe, dans les sphères politiques et intellectuelles, qu'une entreprise parallèle axée sur la recherche d'explications et de légitimation du nouvel "ordre mondial» émerge: une nouvelle "idéologie ", si on veut récupérer cette expression démodée, qui semble plutôt confuse et parfois contradictoire.

Ce ne sont pas toutes les conceptions de la scène mondiale qui sont cosmopolitiques. Il existe en effet de nouvelles versions de l'impérialisme (néo)conservateur et nationaliste qui sont explicitement anti-cosmopolitiques. Ce ne sont pas non plus toutes les conceptions du cosmopolitisme qui adoptent un point de vue moral. Certaines invoquent plutôt l'ouverture d'esprit du voyageur à l'affût de nouvelles expériences, qui peuvent être triviales, mondaines et moralement neutres. D'autres mettent l'accent sur l'intérêt porté à la vie et à la culture d'autrui, et impliquent une tolérance et une volonté d'apprendre qui suggèrent à tout le moins une certaine sensibilité morale et une conscience des différences sociales. Ainsi, sans nier la possibilité que le cosmopolitisme s'accommode d'une indifférence morale - ce que lui reprochent parfois ses critiques - on peut soutenir que l'expérience consistant à être confronté à des différences culturelles a, sur les croyances d'une personne, un effet relativisant. Cet effet peut mener à l'adoption d'une atti-

5. Reiss, 1970, pp. 93-130.

6. Voir Bohman et Lutz-Bachmann, 1997. 
tude réflexive et autocritique qui incite à l'échange de vues sur des questions morales. On pourrait alors dire que le cosmopolitisme, même dans la version purement esthétique où il prône la curiosité intellectuelle et l'abandon de l'esprit de clocher, peut servir d'antidote au dogmatisme bien-pensant. Au regard de la montée de nouvelles formes de fondamentalisme politique et religieux à l'échelle mondiale, c'est une caractéristique du cosmopolitisme ancien que l'on pourrait vouloir préserver.

Il y a néanmoins plusieurs versions contemporaines du cosmopolitisme, comme le montre l'abondance des écrits récents sur ce sujet ${ }^{7}$. Tous ne le voient pas d'un œil favorable. Pour certains de ses critiques, l'apparente ouverture d'esprit professée par le cosmopolitisme masque en réalité une affinité historique interne avec l'impérialisme ${ }^{8}$. Certaines de ces objections seront examinées en détail dans les sections qui viennent; mais avant de nous tourner vers elles, quelques précisions semblent nécessaires. Remarquons d'abord que, dans la foulée d'une opposition à la mondialisation économique dénonçant les nouveaux déséquilibres de pouvoir et les grandes inégalités que celle-ci produit à l'échelle mondiale, plusieurs se montrent extrêmement méfiants envers la totalité du processus de mondialisation ${ }^{9}$, de même qu'envers les diverses tentatives qui visent à définir la justice à l'échelle mondiale ${ }^{10}$. D'un côté comme de l'autre, cependant, la question des différences culturelles apparaît comme un problème central. Un changement, dans le décor contemporain de ce débat, vient du fait que la rencontre avec «l'autre », en termes culturels, n'est plus quelque chose d'inhabituel; elle est plutôt devenue une partie de notre expérience quotidienne. L'immigration et l'émergence de nouvelles communautés ont changé le visage de lieux qui étaient auparavant homogènes sur le plan du langage et de la composition ethnique. L'augmentation du nombre des voyages effectués pour toutes sortes de raisons, les médias de masse et leur appropriation des us et coutumes des peuples étrangers pour la publicité et l'industrie du divertissement (jusqu'à ce que «l'exotisme» soit devenu lieu commun), de même que l'accès généralisé à l'information, ont créé de nouvelles formes d'identification et d'organisation sociales. C'est le cas, en particulier, des communautés d'immigrants et des organisations civiles supranationales. Tout cela, en retour, a eu de profonds effets sur les identités culturelles et a amené tout un nouvel ensemble de problèmes économiques et politiques.

Dans l'arène politique, et même si nous ne voulons pas nous engager ici dans la polémique sur la mort des États-nations, il est indubitable que ces derniers ont connu des changements internes et que leur identité culturelle est en voie de redéfinition. Ils peuvent être réorganisés en unités politiques et

7. Comme en fait état, par exemple, David A. Hollinger.

8. Pagden.

9. Voir, par exemple, Brennan, 1997.

10. À titre d'exemples de telles tentatives, voir entre autres Held, McGrew et al, 1999; Nielsen, 2003 ; María Xosé Agra et al., 2002. 
économiques plus vastes, comme dans le cas de l'Union européenne, ou encore modifiés de quelque manière quant à leur structure traditionnelle. Aux yeux de certains auteurs, comme Ulrich Beck ${ }^{11}$, il y a là des implications importantes pour la méthodologie des sciences sociales: puisque l'État-nation a cessé d'être une unité d'analyse adéquate, de nouveaux outils méthodologiques sont nécessaires pour pouvoir tenir compte des réseaux d'interdépendance allant au-delà des frontières nationales. Mais cela n'est qu'un aspect du problème, car la complexité de la situation, instable et dans un processus de changement et de réajustements constants, ne permet pas que l'on s'en tienne à une seule approche. Ce que l'on nomme cosmopolitisme pourrait en fait référer à un certain nombre de problèmes différents. Ainsi, les questions de différence culturelle ne doivent pas être confondues avec les problèmes d'inégalité matérielle, même si certaines relations devaient être établies entre elles. Dans chacun de ces domaines, aussi bien des questions politiques que légales ou morales peuvent tour à tour exiger notre attention. Cela n'est certainement pas la même chose de penser à l'expérience de l'immigration forcée - à cause de la guerre ou de la pauvreté - que de considérer, par exemple, à l'autre extrême, les problèmes auxquels font face les voyageurs - professionnels et gens d'affaires - susceptibles de vivre pendant de longues périodes en pays étrangers et dans l'espace artificiellement homogène de la civilisation technocratique. Dans le premier cas, les communautés nouvelles et relativement isolées peuvent sembler conservatrices en ce qu'elles développent des attitudes défensives qui compensent en fait pour l'exclusion et le rejet auxquels elles font face dans leur nouvel environnement (donnant ainsi l'impression d'être plus «traditionalistes » qu'elles ne le sont ou faisant de la «différence» une bannière politique). Dans le deuxième cas, les groupes peuvent sembler plus progressistes en un sens politique, alors qu'en fait ils tiennent simplement pour acquise la supériorité de la culture dominante à laquelle ils appartiennent et la légitimité incontestée de leur statut privilégié. Et, bien sûr, à l'intérieur de ces groupes, ainsi que dans d'autres, possiblement touchés par des déplacements transculturels, on peut trouver à la fois des positions progressistes et des positions conservatrices. La tendance à assigner une connotation politique ou morale unilatérale à ses adversaires, que ceux-ci soient des particularistes (communautaristes, traditionalistes ou nationalistes) ou des défenseurs du cosmopolitisme (universalistes moraux ou pluralistes culturels), est un aspect particulièrement insatisfaisant du débat qui les opposent.

On peut donc soutenir dès le départ qu'il n'est plus possible de caractériser le débat opposant le cosmopolitisme au particularisme comme une confrontation entre une conception du global en tant qu'il impliquerait nécessairement une conception politique, légale ou morale unifiée, et une conception du local (qu'il s'agisse de communautés ou de nations) en tant qu'isolé ou 
autonome. Il n'est pas non plus souhaitable d'essayer de couvrir trop d'aspects à la fois de ce champ problématique. Il semble plus productif d'essayer d'abord d'explorer certains modes spécifiques par lesquels les entités locales transformées sont reliées à différentes formes d'intervention globale et de tenter ensuite de voir comment cela affecte nos théories. Aux fins de cet article, il sera important d'examiner les façons dont une certaine conception minimale de la justice pourrait être acceptée et adoptée du point de vue de ces particularités modifiées que sont les identités personnelles contemporaines. Du point de vue des personnes concernées, la question devient celle de trouver des raisons d'adopter des principes moraux et de suivre des normes dont la portée va bien au-delà de leur situation existentielle particulière sans toutefois aller à l'encontre des intérêts reliés à tous ces aspects de leur vie susceptibles de faire l'objet de revendications légitimes. J'examinerai aussi la question de la responsabilité morale dans le nouveau contexte d'un espace public mondial. Je n'analyserai pas toutes les différentes versions du particularisme et du cosmopolitisme qui ont pu être proposées, mais je suis d'accord avec certains auteurs sur le fait que le débat vise avant tout, d'une part, à répondre à une vieille accusation voulant qu'on ait négligé l'importance du particulier, et, d'autre part, à établir des distinctions entre les différentes versions du cosmopolitisme ${ }^{12}$. Ce sont ces questions que j'aborderai dans la prochaine section.

\section{Cosmopolitisme et différence culturelle}

On a souvent reproché aux Lumières un universalisme vide et abstrait, trop détaché des réalités de l'existence quotidienne. On a aussi dénoncé leur appel naif à une humanité commune, qui ne tient pas compte des luttes de pouvoir ou des différences culturelles radicales ${ }^{13}$. Ces deux objections ont été soulevées à propos de toutes les formes d'universalisme moral, et non pas seulement à propos du cosmopolitisme. On peut les interpréter comme soulignant la présence d'un conflit entre, d'une part, les affiliations locales et les raisons liées aux intérêts privés des agents et, d'autre part, les raisons publiques qui font appel aux devoirs envers l'humanité ou à la solidarité mondiale, ou bien entre deux codes moraux différents et parfois incompatibles (privé et public, religieux et civique, local et cosmopolite). Mais de tels conflits sont une caractéristique générale de l'expérience morale; ils ne sont pas exclusifs au cosmopolitisme. Nous sommes souvent confrontés à des décisions qui nous forcent à choisir entre des exigences concurrentes posées par les codes de conduite respectifs de nos différents champs d'expérience. Les critères que nous utilisons pour décider de ce qui doit avoir préséance dans une situation donnée ne sont, bien sûr, ni fixes ni stables. La question du conflit moral

12. Hollinger.

13. Comme on l'a aussi reproché à Martha Nussbaum dans Cohen, 1996. 
fait l'objet d'un débat ouvert en théorie morale, et nous ne pouvons nous intéresser ici qu'à certains de ses aspects.

\section{1}

J'examinerai la question du conflit, soi-disant inévitable, entre, d'une part, l'affiliation locale et la loyauté qu'elle implique envers la famille et, d'autre part, la communauté et une allégeance de type cosmopolitique. Ce conflit peut survenir sous des formes plus complexes que celles qui sont normalement reconnues. Sa forme habituelle oppose au cosmopolitisme l'idée de la primauté des responsabilités envers notre communauté ou encore, dans le cas du nationalisme, envers nos compatriotes. Mais de plus en plus s'impose l'exigence d'un respect envers les particularités culturelles de communautés qui sont en quelque manière marginales par rapport à l'identité dominante au sein d'un État-nation. Dans le premier cas, nous restreignons la portée de nos devoirs légaux et moraux à l'ensemble des individus placés sous la juridiction d'un État-nation. Dans le second, nous ajoutons une exigence plus fondamentale: celle de rendre possible la poursuite d'une vie normale, incluant le respect des devoirs moraux envers nos compatriotes, dans des conditions de liberté et de respect d'autrui. Dans les deux cas, l'hypothèse de départ est que les premiers devoirs moraux sont attachés, ou limités, aux "communautés de sentiment ", en vertu de ce que des auteurs tels que Henry Shue ont appelé « une morale en cercles concentriques ${ }^{14}$. Ce point de vue suppose non seulement qu'il est peu plausible psychologiquement d'imaginer des obligations qui vont au-delà des cercles intérieurs des attachements premiers, mais aussi que le paramètre adéquat pour délimiter l'étendue de ces devoirs est l'État-nation. Or l'existence même de communautés marginales (qu'elles soient ethniques, religieuses ou autres) infirme cette dernière supposition. Comme Shue le remarque: "Les attachements primordiaux s'expriment souvent envers des groupes plus petits que les États-nations ${ }^{15}$.» De plus, ces loyautés basées sur les sentiments peuvent s'étendre au-delà des frontières de l'État-nation et revêtir diverses formes de solidarité. Elles peuvent aussi se manifester dans des organisations de la société civile, sollicitant divers types d'engagements, différents de ceux que décrivent les conceptions traditionnelles de la communauté.

Un reproche fréquemment adressé aux défenseurs du cosmopolitisme est qu'ils font fi du besoin d'appartenance et négligent l'importance du rôle que joue une communauté stable dans le développement d'une identité personnelle saine et dans la poursuite d'une vie pleinement vécue. Ce reproche vient tout autant des communautariens que des défenseurs de la primauté de l'identité locale (ou nationale) vis-à-vis des exigences morales plus abstraites ou générales. Mais cette critique semble tenir pour acquis une stabilité et une homogénéité des identités culturelles bien plus grandes que celles qu'on peut observer de nos

14. Shue, 1980, pp. 144-152.

15. Ibid., p. 145. 
jours dans la plupart des sociétés. Jusqu'à un certain point, l'identité nationale est une construction sociale qui procure un certain sentiment de sécurité et offre une représentation de la vie commune tout en façonnant les valeurs et les habitudes. Mais c'est une construction qui ne tient pas compte, dans ses descriptions canoniques, de la présence des autres communautés et identités culturelles qui existent de fait au sein de la nation. Même le lien entre l'attachement territorial et une identité collective n'est plus aussi clair qu'avant, comme en témoignent les nombreuses minorités qui vivent à l'extérieur de leur territoire d'origine. De plus, le besoin de protection légale pour les gens qui vivent dans ces circonstances imprévisibles est plus urgent que jamais. Il ne s'agit pas ici de nier l'importance de ces formes d'affiliation et de loyauté. Il s'agit plutôt d'écarter des idées reçues et des oppositions entre affiliations locales et règles de justice globale qui ne sont plus valides dans le contexte actuel. La défense des valeurs et des coutumes traditionnelles peut être un moyen de contrer une assimilation forcée, mais ce qu'il y a de nouveau dans cette défense des différences culturelles, c'est que maintenant, pour reprendre les mots de Saskia Sassen, ce qui est « local» est aussi global ${ }^{16}$. Il est vrai que les changements rapides dans la vie économique, l'envahissement des sociétés traditionnelles par une culture de consommation globale ou la présence de groupes d'immigrants à l'intérieur des États-nations sont souvent perçus par les communautés locales comme une menace pour leur existence même. Mais il est aussi vrai que l'isolement des communautés n'est plus une solution plausible.

Cette situation affecte également certaines mesures bien intentionnées prise à l'endroit des communautés traditionnelles — comme de reconnaître l'autonomie politique des peuples indigènes ${ }^{17}-$, car ces mesures peuvent avoir l'effet indésirable d'exclure les étrangers et les dissidents, ou d'induire des conflits au sein des communautés lorsque, par exemple, certains de leurs membres partent chercher ailleurs du travail ou de meilleures perspectives d'avenir. Cet exemple illustre la contradiction à laquelle nous exposent de telles mesures: les immigrants exigent légitimement que l'on respecte leur identité collective et leurs droits en tant que groupe, ce qui remet en cause certaines conceptions libérales, mais ils exigent en même temps des protections légales de la part de l'État ou du droit international, ou d'autres institutions internationales libérales ou démocratiques. Ce qu'on peut conclure de ces expériences - celles des immigrants et des autres communautés impliqués dans ce processus - ce n'est pas que les identités doivent être absorbées ou assimilées de force; c'est plutôt qu'elles doivent faire l'objet de négociations politiques complexes, dans de nouvelles conditions d'interactions sociales qui s'écartent du modèle des unités culturelles distinctes et autonomes. De plus, il semblerait que certaines règles de justice de base communes soient nécessaires à une coexistence pacifique des

16. Sassen, 1991.

17. Pour un traitement plus détaillé de ce problème, voir Herrera, 2003. 
communautés dans notre contexte marqué par l'hétérogénéité culturelle et le déracinement.

\section{2}

Mais la question de la préservation des valeurs et des habitudes dans les sociétés touchées par les changements démographiques et par les effets de la mondialisation économique et culturelle requiert aussi une nouvelle compréhension du concept d'identité culturelle. Les façons de faire et les habitudes qui ont cours dans les pays développés de même que les identités personnelles et collectives des immigrants ne restent pas inchangées tout au long de ce processus. Parmi les immigrants, plusieurs ont des réactions mitigées à l'endroit des valeurs traditionnelles de leur communauté; on observe aussi l'émergence de nouvelles formes d'identités culturelles qui laissent supposer un certain dialogue interculturel et un certain apprentissage social.

Le point de vue des émigrés nous permet de mettre en doute l'image que l'on se fait habituellement des communautés fermées et homogènes, et aussi celle du cosmopolitisme tel que le voient les exclus et ceux qui viennent de pays non européens. Ce que Joan Cocks a décrit comme le «cosmopolitisme négatif ${ }^{18}$ de certains immigrants peut nous servir de point de départ. Le cosmopolitisme négatif est caractérisé par la perte de l'innocence et la désillusion des immigrants à l'endroit des croyances et des traits culturels de leur lieu d'origine et par les changements, dans leurs habitudes culturelles et dans leurs valeurs, qui résultent inévitablement de l'apprentissage et de l'adaptation. Dans un article comparant l'expérience d'exil de deux écrivains, elle retrace, dans les ouvrages de l'un d'entre eux, V.S. Naipaul, le désenchantement de l'immigrant. Le point de vue de Naipaul n'est ni nostalgique ni complaisant, et il n'est pas non plus simplement amer. Il s'inscrit plutôt en faux contre les conceptions simplistes de l'identité culturelle. Les personnages de ses romans ne peuvent plus croire à la pureté de leurs façons de faire traditionnelles puisqu'ils peuvent maintenant reconnaître les effets de toutes sortes de métissages ethniques et d'échanges culturels entre les diverses communautés. De plus, ce qui était vu comme ayant une valeur indubitable dans leur lieu d'origine peut faire la risée des membres de la communauté dominante. Il devient donc plus difficile d'avancer et de soutenir une prétention à l'authenticité; et la réaction des immigrants à ces expériences ne peut plus simplement consister à se réfugier dans une culture minoritaire, du moins pour la plupart d'entre eux. L'œuvre de Naipaul met au jour un monde instable, inégal et de plus en plus métissé, qui ne laisse plus de place à une image idéalisée de la communauté.

La question de l'identité culturelle n'est donc pas si claire, et l'expérience de l'exilé pourrait fort bien être celle d'apprendre à vivre dans des conditions instables et changeantes, mais d'une façon qui n'implique pas nécessairement l'abandon de toute allégeance ou responsabilité envers son peuple. C'est une

18. Cocks, 2000. 
réalité que le deuxième auteur étudié par Cocks, Edward Said, rend explicite. Le cosmopolitisme qu'il défend suppose un engagement des immigrants à l'endroit de leur communauté politique; il échappe ainsi à l'objection habituelle voulant que le cosmopolitisme implique le rejet de toute attache locale. Mais Said reconnaît en même temps que l'expérience d'avoir vécu en plusieurs endroits du monde, en contact direct avec les autres, peut avoir pour effet d'approfondir la compréhension d'autrui. Il deviendrait ainsi possible de penser aux autres de façon «concrète et sympathique ", plutôt que de limiter la portée des obligations morales au «nous» communautarien ou nationaliste ${ }^{19}$. En fait, il soutient que l'on peut développer une moralité plus inclusive en réfléchissant aux questions morales du point de vue de différentes pratiques culturelles. Sa "mondialité ", comme l'appelle Joan Cocks, diffère des versions européennes habituelles en ce que, en plus d'adopter les valeurs universalistes des Lumières, elle assume un engagement plus prononcé envers «les pauvres, les désavantagés, les sans voix ${ }^{20}$. C'est un point de vue marginal, mais qui n'est pas déconnecté des réalités de notre monde hybride et multidimensionnel.

Said nous rappelle que la tentative de placer ses propres croyances et valeurs au-dessus de celles de tous les autres et d'élaborer ainsi une forme exclusive du nationalisme a historiquement été la première source de l'impérialisme ${ }^{21}$. Mais, accompagnant ces tentatives, on peut toujours observer des formes de résistances et des mouvements sociaux qui ont finalement réussi à vaincre la domination coloniale. Dans une analyse de la façon dont ces luttes politiques et culturelles en sont venues à être représentées dans la littérature narrative, Said nous présente ainsi le colonialisme comme une interrelation complexe entre deux phénomènes: d'un côté l'image de soi des colonisateurs en tant que représentants d'une culture supérieure qui, à elle seule, justifierait leur conquête, de l'autre, les idéaux de justice universelle amenés par les colonisateurs euxmêmes et qui se sont finalement retournés contre eux.

Said est un auteur intéressant du point de vue des questions que nous posons dans cet article. Premièrement, en raison de ses analyses des relations entre l'Ouest métropolitain et ses colonies et, en particulier, des effets à long terme de la colonisation à la fois chez les peuples colonisateurs et chez les peuples colonisés. Deuxièmement en raison de sa biographie, où il trace de lui-même le portrait d'une identité culturelle complexe. Sur la question des relations entre l'Ouest métropolitain et ses colonies, on peut trouver, dans son œuvre, quelques balises pour une conception non essentialiste de la culture. Il ne s'agit pas seulement, pour lui, d'admettre que l'histoire de la plupart des peuples et des nations est marquée par de multiples interventions et interconnexions avec d'autres groupes et d'autres cultures (immigration, conquêtes, colonisation, etc.). Il faut encore reconnaître, dit-il, que ce qu'on appelle "culture», non seulement en tant

19. Ibid., p. 57.

20. Ibid.

21. Said, 1996.

22. Ibid., pp. 11-34. 
qu'habitudes et pratiques usuelles, mais aussi en tant qu'artéfacts ou en tant que représentations littéraires, est une source potentiellement explosive d'identité nationale. La culture est une sorte de "scène ${ }^{22}$ où des batailles culturelles ont lieu: les positions idéologiques sont confrontées les unes aux autres en tant qu'interprétations rivales de la culture, en tant que formes d'appropriation sélective du passé et en tant qu'annonciatrices des orientations futures. Puisque les colonisateurs ont fourni le langage et les formes narratives de ces interprétations, ils ont, d'une part, façonné leur expérience de la colonisation de façon à justifier l'entreprise coloniale; on en trouve plusieurs exemples dans les romans et aussi dans les traités politiques. Mais, d'autre part, ils ont aussi fourni des récits d'émancipation et d'autres écrits qui ont contribué au processus de libération; un exemple éloquent est l'influence exercée par les Encyclopédistes français sur les mouvements d'indépendance en Amérique latine.

Mais, selon Said, il y a aussi un autre aspect, plus ambigu, de la culture: il s'agit de la culture comme "élévation ». Il n'est pas ici seulement question de la consécration des valeurs et des accomplissements de la culture dominante, mais aussi du déni des pratiques quotidiennes et ordinaires de l'Empire: de la cruauté de l'esclavage, des autres abus de l'expansion coloniale et des façons de faire de son administration. Tout cela était exclu de la sphère transcendante de la haute culture et appartenait à un champ séparé. En dépit de l'existence d'informations suffisantes sur ces pratiques (registres officiels et autres), elles ont été ignorées par les intellectuels et les artistes (et par les philosophes), et aucune connexion systématique n'a été établie entre la culture et cette réalité. Ainsi, pour les peuples qui ont un passé colonial - en fait, un bon nombre des peuples du monde non inclus dans l'Europe moderne - il y a une ambivalence fondamentale à l'endroit des retombées culturelles des centres métropolitains: elles peuvent faire l'objet d'une admiration esthétique et intellectuelle, mais elles ne peuvent nourrir d'illusions quant à leur innocence politique. Pour Said, le projet de conquête globale de l'impérialisme est intrinsèquement lié à l'histoire des peuples colonisés, mais ce lien n'apparaît pas dans une histoire officielle unifiée. Plusieurs fragments de l'histoire de ces peuples pourraient être reconstitués en faisant place au point de vue des exclus: certains sont déjà documentés, d'autres attendent de l'être. C'est précisément ce genre de connexions que Said cherche à rétablir dans son œuvre en évitant de faire de la culture une sorte de "protection » contre les réalités déplaisantes du pouvoir et de l'oppression.

Mais ce qui distingue l'approche de Said des autres approches postcoloniales ou postmodernes tient à ce qu'il ne rejette pas la possibilité d'une conception universelle de la justice. Certes, selon lui, la base d'une telle conception ne réside pas dans une hypothétique égalité de l'humanité rationnelle transcendant les différences de race, de nationalité, de religion, comme le voulait la formule classique des Lumières. C'est que pour Said, en fait, cette vision des choses inclut, sans les reconnaître, les traits de la culture européenne (et maintenant américaine) qui ont servi à la justification culturelle de la domination impériale. Les 
seules bases possibles, selon lui, d'une conception de la justice cosmopolitique doivent plutôt être trouvées dans l'agrégation et le mélange des cultures et des différentes formes de vie, et prendre en considération les écarts de pouvoir et de bien-être matériel qui existent présentement dans les sociétés qui en sont porteuses. Sa conception de la justice est ainsi une version de l'universalisme moral en tant que somme des différences, qui met de l'avant une certaine forme d'égalité fonctionnelle, dont l'énoncé précis reste à formuler mais qui n'est pas incompatible, en principe, avec une certaine idée des droits universels de base. Finalement, la procédure qu'il suggère pour résoudre les conflits potentiels entre l'expérience morale vécue dans les sphères privées ou locales et les exigences de la moralité universelle ne consiste pas à accorder la priorité absolue à ces dernières; puisque ce qui compte pour local et pour universel peut être circonscrit de diverses façons selon les interprétations différentes qu'en donnent différentes cultures, on devra décider au cas par cas et en tenant compte des interprétations culturelles de ce qui appartient à chaque type d'obligation morale.

Plusieurs questions restent néanmoins en suspens parmi lesquelles il faut mentionner les modes de justification d'une justice minimale à l'échelle mondiale, les procédures d'un dialogue interculturel et celles de la résolution de conflits. Mais la façon dont Said aborde la question de l'identité culturelle constitue une réplique intéressante et stimulante aux objections habituellement soulevées contre l'idée d'une justice cosmopolitique. La question est à tout le moins placée dans un éclairage différent qui fait ressortir la nécessité d'un dialogue réel entre les membres de différentes cultures, et d'une forme de réflexion et d'appropriation critique de la culture du point de vue des différents acteurs sociaux. Cette façon de voir les choses évite le déterministe et le dogmatique et trace un portrait plus réaliste de la vie en communauté, qui rompt avec les idéalisations romantiques des communautariens.

\section{Le cosmopolitisme comme impérialisme}

Il y a toutefois une autre objection contre l'universalisme cosmopolitique sur laquelle nous devons nous pencher parce qu'elle lui est adressée depuis ses tout débuts et qu'elle accompagne souvent les accusations d'ethnocentrisme formulées par ses critiques issus des nations non européennes et des régimes postcoloniaux. Cette objection soulève un doute au sujet du cosmopolitisme: même dans ses versions éclairées, serait-il impérialiste?

Il est intéressant de constater la continuité qui existe, de ce point de vue, entre certaines critiques des anciennes formes de cosmopolitisme et ses récentes critiques post-modernes et post-coloniales. Ces critiques soulèvent principalement deux questions souvent jumelées au sein d'un même argument, mais qui sont néanmoins analytiquement distinctes. La première, de nature conceptuelle, porte sur le caractère inclusif ou non de l'ancienne Cosmopolis et, par extension, des autres versions du cosmopolitisme universaliste. En d'autres mots, on soupçonne le cosmopolitisme de ne pas être aussi juste et 
inclusif que ne le prétendent ses défenseurs. La seconde question, de nature empirique, met en cause les usages politiques de l'idée d'un système légal-moral qui voudrait s'imposer comme un modèle unifié pour le monde.

\section{1}

On peut commencer en examinant brièvement les origines de l'idéal cosmopolitique. Comme nous le rappelle Martha Nussbaum, un thème central de la pensée cosmopolitique depuis ses débuts est celui de l'allégeance simultanée à deux communautés, l'une "vraiment grande et vraiment commune, embrassant les dieux et les hommes " par delà les frontières et les particularités, et l'autre, "celle que la naissance nous a assignée » ${ }^{23}$. Dans la Grèce et la Rome antiques, il était déjà possible de concevoir qu'une unité plus grande que la communauté locale puisse être une source d'obligations morales — par exemple, ne pas nuire aux autres communautés ou pratiquer l'hospitalité envers les étrangers - et ce, même en l'absence d'arrangements légaux ou au risque d'aller à l'encontre des pratiques de la vie politique locale. Cela impliquait, pour les communautés locales, de se percevoir comme parties d'une humanité rationnelle commune et comme membres d'une «cité ${ }^{24}$ plus vaste, mondiale. Ce sont ces idées, plutôt que certains aspects spécifiques des buts institutionnels et pratiques qu'auraient pu poursuivre leurs défenseurs, que Nussbaum considère comme étant au cœur du cosmopolitisme classique. Ce sont elles encore, toujours selon Nussbaum, qui ont exercé une grande influence sur la philosophie morale de Kant et que celui-ci a reformulées dans ses principes de respect universel et de justice cosmopolitique ${ }^{25}$.

Un doute quant à l'influence de ces idées sur les pratiques réelles peut toutefois être soulevé dès le départ: le monde était-il vraiment conçu, dans la pensée antique, comme formant une seule cité, régie par une même règle de justice et où tous ont une même valeur? Dans un article où il soulève ces questions, Anthony Pagden soutient le contraire, affirmant que «les stoïciens, en effet, loin d'embrasser tous les peuples, semblent n'avoir regardé comme dignes de leur considération que ceux qu'ils tenaient pour sages ${ }^{26}$ ». En outre, plutôt que de considérer l'humanité commune comme étant la source d'une radicale égalité de statut, ou comme conférant à toutes les cultures (et à toutes les croyances et coutumes) la même valeur, ce que les stoïciens auraient proposé était plutôt l'expansion de la cité grecque; cela voulait dire étendre la polis grecque à (certains) non-Grecs, comme le fit la loi romaine par la suite. Ainsi, la visée première des anciens défenseurs du cosmopolitisme aurait été l'inclusion inconditionnelle de tous dans leur système moral ou, plus précisément, la négation des valeurs et des croyances d'autrui. Plutôt que d'être vu comme une affirmation de la pluralité des peuples, leur cosmopolitisme pourrait être vu comme une ten-

23. Sénèque, cité par Nusbaum, 1997, p. 25.

24. Nussbaum, 1997, p. 33.

25. Reiss, 1970.

26. Pagden, p. 5. 
tative de les assimiler et de leur imposer leurs propres standards et habitudes culturelles - une objection fréquemment soulevée de nos jours à propos de diverses missions « civilisatrices ».

Supposer la validité inconditionnelle de ses propres modes de vie et tenter de les imposer à d'autres, comme l'ont fait les empires grecs et romains, sont deux choses bien différentes. Dans le premier cas, on suppose l'existence d'une relation interne entre raison et justice; c'est la base théorique de l'universalisme moral. Dans le second, on fait un usage politique d'un système de croyances pour justifier des conquêtes militaires. Cet usage est politiquement questionnable et peut fort bien entrer en contradiction avec la présumée nature rationnelle des croyances mises de l'avant. Le premier cas repose sur une affirmation de la condition naturelle des être humains comme créatures rationnelles, au-delà des particularités de leur situation existentielle. Comme le remarque Cicéron:

Toutes les créatures ayant reçu de la Nature le don de la raison ont aussi reçu la droite raison, et elles ont donc reçu aussi le don de la loi, qui est la droite raison appliquée aux directives et aux interdictions. Et si elles ont reçu la loi elles ont reçu la justice. Or tous les hommes ont reçu la raison, donc tous les hommes ont reçu la justice ${ }^{27}$.

C'est la principale leçon que l'on doit retenir des premiers défenseurs du cosmopolitisme. Toutefois, ce n'est pas uniquement la nature rationnelle commune qui a motivé la réflexion des stoïciens. Cela est clair dans le cas de Sénèque, qui a vécu en exil, a eu une vie aventureuse et dont les écrit sont tout autant inspirés d'une réflexion sur ses propres expériences que des enseignements des anciens philosophes. La leçon à retenir, en fait, pourrait bien inspirer une critique du rationalisme abstrait qui fonde les versions ultérieures de l'idée de justice universelle. Dans les formulations anciennes, il n'y avait pas de hiatus entre la réflexion philosophique sur la nature rationnelle de l'homme d'une part, et d'autre part l'expérience affective et la motivation morale. Un passage souvent cité de Sénèque est tiré d'une lettre de consolation qu'il adressait à sa mère et qui est, en fait, une réflexion sur l'exil: "Alors, le pas ferme et la tête droite, hâtons-nous là où nous mèneront les circonstances et parcourons tous ces pays, quels qu'ils soient: il ne peut y avoir de lieu d'exil en ce monde puisque rien de ce qui s'y trouve n'est étranger à l'homme ${ }^{28}$.» Dans la même lettre, il y a nombre de réflexions sur la vie des immigrants et sur les changements survenus dans le monde à la suite de l'expansion de l'Empire romain, mais qui sont présentés comme existant déjà depuis longtemps: " [...] Portez votre attention sur les affaires humaines et vous verrez que de nombreux peuples et nations ont changé de demeure. Que font des cités grecques en plein cœur des territoires barbares? Pourquoi entendons-

27. Ibid., pp. 5-6.

28. Seneca, 1997, pp. 11-12.

29. Ibid., p. 9. 
nous parler la langue macédonienne parmi les Indiens et les Perses ${ }^{29}$ ? » Et sur les immigrants: «Certains d'entre eux, entraînés dans une longue errance, n’ont pas délibérément choisi leur but, mais se sont installés par épuisement dans l'endroit le plus rapproché, d'autres par la force des armes ont établi leur droit en pays étranger ${ }^{30}$.» Les changements dont il est question ici se sont produits pour toutes sortes de raisons, des désastres naturels aux guerres, en passant par la condamnation à l'exil dans le cas personnel de Sénèque. Mais ce qui était clair pour lui, c'est que le monde changeait constamment et que l'homme avait besoin d'une certaine forme de compensation pour la souffrance et les épreuves que ces changements lui infligeaient. Et cette conviction lui venait de la croyance selon laquelle "d'où que l'on vienne, on doit composer avec le même ordre naturel» et aussi de cette autre, selon laquelle on doit se fier à la conception de la justice des exilés parce qu'ils «portent avec eux leurs propres vertus ${ }^{31} »$. Un regard plus nuancé sur la conception stoïcienne de la justice nous permettrait sans doute de voir qu'elle propose certaines formes d'adaptation à des circonstances nouvelles ou difficiles plutôt que de prôner le détachement complet vis-à-vis des particularités de l'existence ou plutôt que de nourrir les visées expansionnistes de l'Empire.

Quoi qu'il en soit de cette question, on doit reconnaître que la force des idées cosmopolitiques et l'attrait qu'elles ont exercé au cours de l'histoire, et tout spécialement durant les périodes de redéfinition de frontières nationales et de tensions entre des peuples et entre leurs cultures, reposent sur une intuition profonde: celle d'une humanité commune comme source fondamentale d'obligations morales. C'est aussi l'idée qui justifie, de nos jours, les droits de la personne ${ }^{32}$. Et cette intuition ne fait pas exclusivement appel à notre nature rationnelle, mais aussi à une destinée commune, à une interdépendance de fait et au besoin de communication et de coopération sociale qui s'ensuit. Ces thèmes peuvent aussi être retracés dans la plupart des versions de la pensée cosmopolitique et, contrairement à ce qui a parfois été affirmé, ils ne génèrent pas nécessairement un conflit irrémédiable entre les formes locales d'appartenance communautaire et un engagement envers l'humanité. Ils impliquent plutôt, comme Martha Nussbaum le suggère dans ses remarques sur l'idée stoïcienne de "citoyen du monde", que "chacun de nous habite en effet deux communautés: la communauté locale de notre naissance et la communauté des débats et des aspirations humaines ${ }^{33}$ ». Cette conception de la relation du particulier au général par le biais d'autres particularités, qui correspond à ce qui se produit normalement dans les situations empiriques, est au cœur du cosmopolitisme,

30. Ibid.

31. Ibid., p. 11.

32. Ainsi que des mesures visant à garantir la sécurité et la subsistance, comme le propose Henry Shue.

33. Nussbaum, 1997, p. 29. 


\section{2 - Philosophiques / Printemps 2007}

et elle pourrait inspirer une solution intéressante aux conflits qui nous semblent sans cela insolubles entre ces deux dimensions de l'expérience morale.

On objecte aussi à l'idée d'adopter les valeurs d'autres peuples que cela nous conduirait à l'inconsistance des systèmes de valeur (une version du relativisme moral) ou encore, comme le craint Anthony Pagden, à condamner toutes les valeurs à l'insignifiance: en embrassant toutes les valeurs, on finirait par ne plus en avoir du tout. "Aussi fort qu'on puisse le souhaiter on ne peut être à la fois Perse et Athénien ${ }^{34}[. .$.$] .» Bref, on ne pourrait être affilié$ qu'à une seule polis particulière. Nous pouvons répondre à cette objection en attirant l'attention sur notre expérience contemporaine des identités mixtes (culturelle, ethnique et religieuse). Cette expérience montre en effet que non seulement nous pouvons appartenir à plus d'une culture, mais que nous appartenons effectivement à plus d'une culture; que notre identité d'origine peut être altérée de toutes sortes de façons, à cause des influences externes et des aléas de la vie (de l'émigration à l'exil, aux voyages et autres expériences) et aussi à cause de changements délibérés dans nos attitudes et nos valeurs. Il ne s'agit pas de prétendre que tout coexiste toujours dans l'harmonie; comme il a été suggéré plus haut, notre situation rend l'expérience morale ordinaire plus complexe, mais pas impossible. La délibération avec soi face aux exigences rivales peut être menée à bien, tout comme peut l'être un véritable dialogue avec les autres. Ce qui distingue les défenseurs du cosmopolitisme est une volonté d'apprendre hors des contraintes autoritaristes ou dogmatiques, et non pas un improbable rejet de toute forme d'affiliation. Pour ce qui est de la question de l'inclusion, on peut souligner que le cosmopolitisme - distinct en cela de certaines autres versions de l'universalisme moral - fait du respect envers les autres un devoir, même si cela a été exprimé de différentes façons au cours de l'histoire. Comme principe politique (et légal-moral) et comme guide pour l'action, le cosmopolitisme s'est adapté aux circonstances spécifiques où il a été appliqué. En fait, un examen attentif de son histoire permet de voir que le cosmopolitisme réfère bien moins à une doctrine unifiée qu'à un ensemble de conceptions politico-morales possédant certains traits distinctifs et qui doivent être comprises à la lumière de leur contexte historique.

\section{2}

Un des traits distinctifs de la conception moderne du cosmopolitisme est l'idée de tolérance qui lui est associée. On considère habituellement que cette idée s'est imposée en tant que solution rationnelle aux conflits qui ont opposé les confessions religieuses à la suite de la Réforme. Toutefois, elle a d'autres précédents historiques en Europe et ailleurs dans le monde. Par exemple, elle a inspiré

34. Pagden, p. 4.

35. Pendant la dynastie des Omeyas (756 à 1030). Voir à ce sujet Fetscher, 1994, p. 72.

36. Spinoza, 1986. 
la coexistence relativement pacifique des musulmans et des juifs dans l'Espagne médiévale ${ }^{35}$ avant d'être développée par Spinoza ${ }^{36}$ et dans les textes des Lumières. Dans sa version moderne, l'idée de tolérance était déjà une réponse au dogmatisme de l'Église et à son caractère impérial. L'idée de tolérance est aussi associée à l'absence de certitude quant à ses propres croyances; comme le disait Voltaire dans son célèbre essai ${ }^{37}$ : les autres peuvent avoir raison ${ }^{38}$. S'il s'agissait d'abord de s'opposer au fanatisme religieux, les avocats de la tolérance ont jusqu'à nos jours aussi vu celle-ci comme une règle pour une coexistence pacifique et civilisée. Tolérer la différence n'est pas incompatible avec le fait d'avoir de fortes convictions morales et politiques, soutenues par de bonnes raisons, mais cela implique que l'on reconnaisse les imperfections de notre raison pratique; que l'on reconnaisse qu'il peut exister de meilleures façons de traiter certains aspects de l'existence ou de réguler les conflits sociaux, et que ces meilleures façons de faire peuvent venir des autres.

Pour que le cosmopolitisme soit une forme plausible d'universalisme moral, il faut aussi y adjoindre une clause passablement exigeante d'égalité. Il ne suffit pas de postuler une commune nature rationnelle puisqu'il existe aussi des besoins communs, tels que le besoin d'hospitalité qui suppose le gîte et le couvert de même qu'un accueil cordial, dépourvu d'hostilité. La satisfaction de ces besoins requiert bien davantage que ce qu'exigerait la protection minimale contre l'agression inscrite dans la loi locale sur le juste traitement des étrangers. Elle requiert, en fait, d'étendre la portée des obligations qui prévalent dans notre communauté de façon à y inclure les autres. Cette inclusion n'est pas de nature "impérialiste » dans la mesure où elle implique le dialogue et exclut toute forme de violence. Néanmoins, selon Pagden, il serait possible de trouver dans toutes les versions du cosmopolitisme qui vont des stoïciens aux Lumières, ce qu'il identifie comme étant la composante impérialiste de l'universalisme cosmopolitique, à savoir l'idée d'une "communauté des sages ", possédant un caractère exemplaire justifiant non seulement ses décrets d'exclusion, mais aussi la promotion et la diffusion de ses valeurs. Le lien établi ici entre le caractère exemplaire d'un système moral-légal et l'autoritarisme mérite d'être examiné de plus près.

Pour Pagden comme pour d'autres critiques, il serait inutile d'essayer de dissocier la doctrine des Lumières de l'impérialisme - sauvegardant ainsi, en quelque sorte, sa composante universaliste - puisque l'histoire de la philosophie européenne est inséparablement liée à l'idée même du cosmopolitisme. Et le problème ne ferait que s'aggraver dans les conceptions modernes qui endossent un idéal de développement et de progrès moral. L'affirmation de la supériorité des conquérants n'est pas le propre des impérialismes européens,

37. Voltaire, 1976, p. 112.

38. Et cela signifie en retour que la tolérance elle-même n'est pas qu'un héritage de la culture européenne. C'est une tâche qui reste à faire que d'étudier les idées de liberté, de justice et d'égalité dans d'autres cultures. Pour une contribution, voir Sen, 2001. 


\section{4 - Philosophiques / Printemps 2007}

mais ce qu'il y a de particulier à l'histoire européenne est le type de justification idéologique associé à cette affirmation, c'est-à-dire, la nature de cette prétention à un statut culturel supérieur. Cette prétention, en effet, s'appuyait sur la thèse d'un développement graduel, progressif, des capacités rationnelles des peuples européens et de l'état "d'achèvement » de la civilisation européenne, qui se sentait dès lors le droit d'étendre partout sa suprématie, comme si c'était le cours naturel de l'histoire.

On peut en effet trouver des éléments de cette croyance dans la philosophie des Lumières, ainsi que dans la justification de l'Empire britannique par Locke, et même chez Kant, malgré sa critique du colonialisme. Ce qu'il faut se demander ici, c'est de quelle façon ces deux aspects antagonistes de la philosophie pratique de Kant sont liés entre eux. La question qu'il importe de soulever, comme le remarque Thomas McCarthy, est celle de savoir comment la notion de développement peut s'insérer dans la tentative kantienne visant à «réconcilier l'universalisme moral-politique avec le particularisme empirique-historique ${ }^{39}$. De plus, ce ne sont pas seulement la téléologie naturelle et l'idée du développement des capacités rationnelles des espèces, comme elles apparaissent dans «Idée d'une histoire universelle au point de vue cosmopolite ${ }^{40}$, qui risquent de produire ce que McCarthy appelle des "dissonances cognitives". Ce sont les propos de Kant sur les races et sur le statut inférieur des non-Européens qui, encore plus, sont clairement incompatibles avec sa philosophie morale universaliste. McCarthy demande donc, prenant argument du caractère systématique allégué de la philosophie pratique de Kant: «Le modèle diffusionniste du progrès, y compris l'idée d'une mission civilisatrice - même si celle-ci n'est pas toujours admise explicitement - est-il compatible avec un monde à venir où ceux qui bénéficient passivement du développement seront sur un pied d'égalité culturel, politique et moral avec ceux qui en sont à l'origine ${ }^{41}$ ?» En d'autres mots: comment les inégalités de source biologique entre les peuples du monde, présupposées par la théorie du développement de Kant, peuvent-elles être conciliées avec les présupposés égalitaristes de son idéal cosmopolitique? Nous ne pouvons certainement pas discuter de tout cela en détail ici, mais nous pouvons néanmoins mentionner que certaines de ces dissonances restent présentes dans bien des conceptions libérales contemporaines. Par exemple, lorsque des États libéraux et démocratiques engagés dans des interventions militaires s'attribuent, au delà de toute vraisemblance, des motifs altruistes ou encore lorsqu'on tient pour acquis les effets nécessairement "progressistes» de la mondialisation capitaliste ${ }^{42}$.

39. McCarthy, 2005.

40. Reiss, 1970, pp. 41-53.

41. McCarthy, 2005. Voir aussi T. McCarthy, «From Modernism to Messianism : Liberal Developmentalism and American Exeptionalism» (manuscrit non publié).

42. Pour une critique de ce point de vue, voir Stiglitz, 2002. 
Ainsi, tandis que les stoïciens ont été critiqués pour l'élitisme qui entache leurs conceptions - n'était pas qui voulait citoyen de la communauté morale du monde - le cosmopolitisme des penseurs des Lumières semble être déchiré par une tension interne. D'une part, dans ses différentes versions, l'idée d'une loi commune aux États reconnaît à ceux-ci une égalité fonctionnelle. Mais, d'autre part, la supériorité culturelle (et morale) de l'Europe n'est jamais mise en doute implicitement ou explicitement. L'objectif même du droit cosmopolitique, à savoir, la paix entre États, étend donc, rhétoriquement parlant, la constitution domestique des États libéraux à toute la planète, tout en cachant ou en ignorant les relations de pouvoir inégales et les ambitions de domination coloniale. Et ce n'est pas seulement que les côtés sombres de l'histoire ont été ignorés; c'est plutôt que certains de ces écrits étaient, de fait, des tentatives de les justifier, indirectement ou pas, en élaborant ce que McCarthy a nommé les «récits magistraux du développement» qui ont continué à se propager tout au long du dix-neuvième siècle, jusqu'à se prolonger, au vingtième siècle, dans les théories sociologiques et politiques de la modernisation.

Nous pouvons donc revenir à une des questions initiales de cet article: est-il possible de défendre une certaine idée de la démocratie et des droits de la personne sans engendrer le soupçon de le faire pour des motifs intéressés ? À quel genre de conditions un système de droits moraux-légaux cosmopolitique doit-il satisfaire pour être vraiment universel et pour recueillir l'assentiment général ? Répondre à ces questions représente un défi pour les défenseurs contemporains du cosmopolitisme. C'est un défi pour la théorie, qui ne peut plus ignorer l'histoire de ses propres implications, qui vont de la justification de la violence du colonialisme à celle des inégalités contemporaines. Et c'est aussi un défi pour la pratique, c'est-à-dire, pour la formulation et l'implantation de normes générales et d'institutions transnationales, telles que les Nations Unies, la Banque mondiale, le Fond monétaire international, qui devront faire la preuve de l'équité de leurs politiques et justifier d'une quelconque façon, même si cela sera toujours sujet à controverse, la distribution inégale du pouvoir et de l'autorité entre leurs membres.

L'inégalité structurelle des institutions internationales fait paraître injustes aux yeux de certains les propositions pour une justice mondiale. C'est le cas de Danilo Zolo qui critique de ce point de vue les travaux des «nouveaux cosmopolites » comme Ulrich Beck, David Held ou Jürgen Habermas ${ }^{43}$. L'une de ses cibles est la proposition, formulée par certains de ces auteurs, de restreindre drastiquement (ou même de rejeter) la souveraineté nationale. Il y a, en effet, de bonnes raisons de défendre la souveraineté nationale pour ce qui a trait aux affaires intérieures et aux politiques publiques des nations plus petites et moins puissantes. Dans des domaines tels que l'éducation, la santé et le contrôle des ressources naturelles, une de ces raisons pourrait être de com- 


\section{6 - Philosophiques / Printemps 2007}

penser pour l'inégalité de pouvoir et de statut qui affecte ces nations. Cette raison serait la version contemporaine de celle qui a été invoquée au début de la modernité pour défendre la souveraineté. Dans ce dernier cas, la menace venait des pays voisins, et les frontières devaient être sécurisées. La menace, dans la situation internationale contemporaine, vient des règles inégales et injustes qui régissent le commerce et le développement économique, ainsi que de l'énorme déséquilibre dans la distribution des ressources et dans l'accès aux instances décisionnelles mondiales. Dans ces conditions, la souveraineté nationale peut parfois être la seule protection contre les abus extérieurs. Nous pourrions bien ne jamais réussir, conformément à l'idéal kantien, à mettre fin à toutes les guerres. C'est ce que nous réalisons une fois que nous avons compris et reconnu la place qu'occupent les relations de domination et les conflits dans l'histoire mondiale. Pour cette raison, nous devons aussi trouver des mécanismes de compensation pour les inégalités. C'est là une modeste aspiration à une forme, certainement imparfaite, de coexistence pacifique entre les peuples.

Ce ne sont pas, néanmoins, toutes les propositions pour une justice globale minimale qui perpétuent nécessairement les vieilles structures impérialistes de relations de pouvoir et les justifications idéologiques qui leur ont été associées. Dans ses écrits récents, Habermas a souligné l'urgence de dissocier l'universalisme moral des conceptions impérialistes qui veulent faire des valeurs particulières qu'elles avancent un modèle pour le reste du monde. Cette exigence est devenue plus explicite encore à la suite des événements récents, comme la guerre contre l'Irak, et l'a amené à souscrire à une conception plus clairement cosmopolitique - aux sens politique et culturel — de la justice universaliste. Il a soutenu qu'un universalisme égalitariste, avant même d'énoncer les exigences formelles d'une justice procédurale, doit commencer par relativiser notre propre perspective de façon à y inclure les interprétations qu'en font les autres, qui doivent être vus comme des égaux ${ }^{44}$. La question de savoir si cela implique une révision de certains aspects cruciaux de son universalisme moral en est une que nous ne pouvons pas aborder ici, sinon pour souligner qu'il semble bien s'agir là, en réponse à ses critiques, de l'annonce d'une reformulation plus politique et moins rationnaliste de l'idée de justice. Quoi qu'il en soit, nous ne pouvons certainement pas espérer lever tous les doutes quant à la faisabilité d'accords généralisés sur les questions de justice. Nous pouvons néanmoins conclure avec quelques remarques sur les conditions qui pourraient faire de ces accords l'objet d'une aspiration plus réaliste.

\section{Conclusions}

La question de savoir s'il peut y avoir une règle de justice universelle capable de recueillir l'approbation de tous ceux qu'elle affecte - une question classique de la philosophie morale - devient plus complexe lorsque la règle en

44. Habermas, 2004, p. 128, et Habermas, 2003, pp. 364-370. 
question affecte potentiellement tous les habitants de la planète. Ce n'est pas seulement que cette question devienne plus radicale, c'est aussi qu'elle implique d'autres problèmes, comme celui de la traduction interculturelle de différents codes et langages normatifs, ou celui d'identifier des raisons que tous les agents peuvent accepter en l'absence de toute coercition. Il y a aussi le problème de l'application des normes, application dont peuvent résulter des sanctions envers des nations ou des peuples vivant dans des conditions d'extrêmes inégalités sociale et économique. Ces problèmes sont à la fois politiques et théoriques, et leur solution ne dépend pas uniquement d'une compréhension légale de l'ordre public puisque la question de la justification morale est intrinsèquement liée à celle de la persuasion. Si l'on pouvait trouver de bonnes raisons, acceptables par les agents particuliers, l'on pourrait aussi obtenir l'engagement de la part de ces agents qu'ils observeront les principes d'une justice mondiale et encourageront leur implantation légale. Contrairement à ce qu'exigerait une compréhension strictement rationaliste du problème de l'acceptation des normes, les raisons acceptables par les agents particuliers pourraient ne pas être les mêmes pour tous. Mais les questions de justification et d'application demeurent toujours enchevêtrées, et les processus d'apprentissage social et moral font toujours intervenir une défense politique des intérêts légitimes, la satisfaction des besoins, ainsi qu'un engagement quant à la moralité. Ainsi, même en prenant comme point de départ la diversité des sujets moraux et de leurs raisons d'être moraux, on peut encore aspirer à sauvegarder l'idéal cosmopolitique kantien d'une "fédération légitime sous un droit international communément accepté ${ }^{45}$.

Mais pour que cela soit possible, nous devons identifier les conditions dans lesquelles pourront être définies des balises minimales en ce qui concerne la coercition illicite et l'imputabilité des agents sociaux et des représentants politiques œuvrant dans les institutions internationales ou supranationales. Sur le premier point, l'usage indu de la coercition, nous pouvons nous inspirer d'un argument avancé par Edward Said ainsi que par les critiques latino-américains de la théorie de la modernisation, à savoir, qu'il y a une continuité entre les asymétries de pouvoir qui caractérisaient autrefois l'impérialisme et celles qui se manifestent maintenant sur le plan économique et militaire. Il importe de nous pencher sur les motifs relevant des intérêts des pays les plus puissants lorsque nous tentons de saisir l'historique et les mécanismes des politiques économiques mondiales et des interventions militaires présumément humanitaires. De plus, ces politiques et interventions ne doivent pas être a priori considérées comme accidentelles ou comme un effet secondaire regrettable des décisions personnelles de politiciens mal avisés. Elles doivent plutôt être vues comme étant liées, d'une façon systématique et programmée, à un agenda de

45. Comme Kant le disait dans sa discussion des coûts de la guerre: «Une distribution inégale des fardeaux ne peut jamais être considérée comme juste. » "Theory and Practice », in Reiss, 1970, p. 90. 
domination. Il est important de se pencher aussi sur le fait que l'enchevêtrement de l'histoire des puissances impériales et de celle des régimes autoritaires (et souvent corrompus) des pays du tiers-monde qui sont leurs alliés est la conséquence d'une structure d'interdépendance. Il sera possible de donner aux pays les moins puissants des raisons convaincantes de restreindre leur souveraineté, en particulier en ce qui a trait aux droits de la personne et aux libertés politiques, lorsque nous aurons établi un droit international et des institutions qui aspirent à tout le moins à être justes et impartiaux et lorsque nous aurons mis en place des mesures susceptibles de limiter l'ambition des pays les plus riches. L'exigence de souscrire aux droits humains et aux libertés politiques doit être vue comme une "condition d'entrée » dans la communauté mondiale des nations. Mais elle doit aller de pair avec un principe d'imputabilité s'appliquant à tous les pays et s'opposant à toute forme d' «exceptionnalisme» comme celui que réclament présentement les États-Unis. L'exceptionnalisme, version agressive de l'impérialisme nationaliste, est aussi menaçant pour une culture généralisée des droits de la personne que les régimes autoritaires et traditionalistes du Tiers-Monde.

On pourrait aussi ajouter qu'un élargissement de la portée de nos responsabilités morales est une conséquence de la nouvelle organisation du pouvoir et des nouveaux mécanismes qui président à la génération et à la circulation de la richesse à l'échelle mondiale. La conception traditionnelle voulant que les obligations morales nous lient uniquement aux membres de notre communauté nationale n'était pas seulement fondée sur l'idée d'un passé commun et d'une identité culturelle commune. Elle s'appuyait aussi sur le fait (allégué) que, le travail et la distribution des biens sociaux ayant une base territoriale, le fardeau des dépenses gouvernementales, des services sociaux et de l'administration de la justice avait aussi des limites territoriales précises. Cette conception de la justice ne réduisait pas celle-ci à une question de solidarité; elle ne se contentait pas non plus d'affirmer la supposée primauté des obligations morales envers nos proches. Elle constituait plutôt une façon raisonnable de conceptualiser la distribution des devoirs et des protections, des bénéfices et des fardeaux matériels. Néanmoins, lorsque le travail de personnes vivant dans une certaine région du monde bénéficie à des personnes vivant dans d'autres pays et que les règles gouvernant les échanges économiques sont systématiquement injustes pour l'une des parties, la conception de la justice distributive doit être revue.

De plus, l'idée de modernité est, encore maintenant, à un point qui n'est pas reconnu dans les versions officielles de l'histoire occidentale, profondément affectée et modelée par les interactions avec les soi-disant non-occidentaux: par l'appropriation de leur travail au sein des territoires coloniaux, par les nombreux «emprunts » à d'autres langages et cultures, et par tous les échanges qui ont eu lieu au cours des cinq derniers siècles. C'est encore un terrain peu exploré par la philosophie et la pensée politique. Nous voulons soutenir, cependant, qu'une culture morale prônant la démocratie et les droits de la personne 
est la seule voie pour atteindre la justice dans la situation présente. Et cela malgré les réserves justifiées à l'endroit de cette culture qui demeure associée à un programme de domination politique et qui n'est pas toujours consistante avec les idéaux qu'elle professe, comme dans les cas d'interventions militaires pour des raisons soi-disant humanitaires. Comme le montre l'exemple paradigmatique des contradictions qui peuvent émerger lorsque le nationalisme (et ses règles d'exclusion) est confronté à la question de l'immigration, et parce que la déstabilisation actuelle des sources traditionnelles de sécurité et d'identité personnelle nous place tous dans une position de vulnérabilité potentielle, des formes de protection légale allant au-delà des frontières nationales sont maintenant nécessaires, de même que le développement de nouvelles formes d'affiliation. Pour ces raisons, certains traits de la moralité universaliste dans sa version cosmopolitique semblent encore souhaitables aujourd'hui.

(Traduit de l'espagnol par Nicolas Payette et Jocelyne Couture)

\section{Bibliographie}

Agra, María Xosé et al. El Derecho de una Democracia Cosmopolita, Anales de la Cátedra, Francisco Suárez, n 36, Universidad de Granada, 2002.

Beck, Ulrich. "The Cosmopolitan Perspective: Sociology of the Second Age of Modernity », The British Journal of Sociology, 51, $\mathrm{n}^{\circ}$ 1, pp. 79-107, 2000.

Bohman, James \& Matthias Lutz-Bachmann, éd., Perpetual Peace, Essays on Kant's Cosmopolitan Ideal, Cambridge, MA, MIT Press, 1997.

Brennan, Timothy. At Home in the World, Cosmopolitanism Now, Cambridge, MA, 1997.

Cocks, Joan. «A New Cosmopolitanism? V.S. Naipaul and Edward Said» Constellations, vol. 7, $\mathrm{n}^{\circ}$ 1, pp. 46-63, 2000.

Cohen, Joshua, éd. For Love of Country, Debating the Limits of Patriotism, Boston, Beacon Press, 1996.

Fetscher, Iring. 1994. La Tolerancia, una Pequeña Virtud Imprescindible para la Democracia, trad. Nélida Machain y Ruth Zimmerman, Barcelona, Gedisa. Traduction de Toleranz, Sttutgart, Radius Verlag, 1990.

Habermas, Jurgen. «Democracia Universal» in Metapolítica, México, vol. 8, nº 35, 2004.

Habermas, Jurgen. «Interpreting the Fall of the Monument », Constellations, vol. 10, $n^{\circ} 3,2003$.

Held, David et A. McGrew et al. Global Transformations: Politics, Economy and Culture, Cambridge, Polity, 1999.

Herrera Lima, María. "On the Rights of Indigenous Peoples: The Case of Chiapas » in Eduardo Mendieta, éd., Latin American Philosophy, Bloomington, Indiana University Press, pp. 165-178, 2003.

Hollinger, David A. « Not Universalists, Not Pluralists: The New Cosmopolitans Find Their Own Way ", Constellations, vol. 8, n 2, pp. 236-248.

Reiss, Hans, éd. Kant Political Writings, Cambridge et New York, Cambridge University Press, 1970.

Kleingeld, Pauline. «Six Varieties of Cosmopolitanism in Late Eigtheenth-Century Germany ", Journal of the History of Ideas, LX, 1999. 
Korsgaard, Christine. The Sources of Normativity, Cambridge, UK, Cambridge University Press, 1996.

McCarthy, Thomas. "Purity and Impurity in Ethics : Kant on Race and Development » dans Razones de la Justicia, María Herrera et Pablo De Greiff, éd., Instituto de Investigaciones Filosóficas, UNAM, México (à paraître 2005).

Nielsen, Kai. Globalization and Justice, New York, Humanity Books, 2003.

Nussbaum, Martha. «Kant and Cosmopolitanism» in Bohman, James \& Matthias Lutz-Bachmann, éd., Perpetual Peace, Essays on Kant's Cosmopolitan Ideal, Cambridge, MA, MIT Press, 1997.

Pagden, Anthony. "Stoicism, Cosmopolitanism, and the Legacy of European Imperialism ", Constellations, vol. $7, \mathrm{n}^{\circ} 1$.

Pogge, Thomas. "Priorities of Global Justice », Metaphilosophy, Special Issue on Global Justice, vol. 32, $\mathrm{n}^{\text {os }} 1-2$, pp. 6-24, 2001.

Said, Edward.1996. Cultura e Imperialismo, Barcelona, Anagrama, traduction de Culture and Imperialism, New York, Alfred A. Knopf, 1993.

Sassen, Saskia. The Global City, New York, London, Tokyo, Princeton, NJ, Princeton University Press, 1991.

Sen, Amartya. «Derechos Humanos y Valores Asiáticos » (XVI Morgenthau Memorial Lecture), in Orden Económico Internacional y Derechos Fundamentales, ACFS, $\mathrm{n}^{\circ}$ 35, Universidad de Granada, pp. 128-147, 2001.

Seneca, Lucius Annaeus. "Consolation to Helvia ", Dialogues and Letters, 8, édition et traduction de C.D.N. Costa, London, Penguin Classics, 1997.

Shue, Henry. Basic Rights, Subsistence, Affluence, and US Foreign Policy, Princeton, NJ, Princeton University Press, 1980.

Spinoza, Baruch. Tratado Teologico-Politico, Madrid, Alianza Editorial, 1986.

Stiglitz, Joseph E. Globalization and its Discontents, 2002.

Voltaire. Tratado de la Tolerancia, trad. Carlos Chies, Barcelona, Editorial Crítica, 1976.

Zolo, Danilo. «¿Una Cosmópolis Imperial ? », Metapolítica, México, vol. 8, n 35, pp. 14-22, 2004. 